\title{
Identification of a Four-miRNA Risk Score Model and Hub Genes as a Novel Potential Prognostic Biomarker in Patients with Glioma
}

\author{
jianguang xu ( $\nabla$ xjg@shutcm.edu.cn ) \\ shanghai university of traditional chinese medicine \\ Si-Si Li \\ Shanghai University of Traditional Chinese Medicine \\ Ye-Chen Lu \\ Shanghai University of Traditional Chinese Medicine \\ Xu-Yun Hua \\ Shanghai University of Traditional Chinese Medicine \\ Jia-Jia Wu \\ Shanghai University of Traditional Chinese Medicine \\ Dao-Fang Ding \\ Shanghai University of Traditional Chinese Medicine \\ Zhen-Zhen $\mathrm{Ma}$ \\ Shanghai University of Traditional Chinese Medicine
}

Research article

Keywords: Glioma, MicroRNA, Hub genes, Prognosis

Posted Date: May 7th, 2020

DOI: https://doi.org/10.21203/rs.3.rs-25457/v1

License: (1) This work is licensed under a Creative Commons Attribution 4.0 International License. Read Full License 


\section{Abstract}

Background Novel biomarkers for malignant glioma are urgently needed due to the poor prognosis of this cancer. Numerous discoveries have demonstrated that microRNAs (miRNAs) participate in the occurrence and progression of glioma. The objective of this study is to explore new biomarkers related to glioma prognosis by bioinformatic analysis.

Methods The miRNA, mRNA expression profiles and relevant clinical information were extracted from TCGA database. Differently expressed miRNAs (DEMs) and differently expressed mRNAs/genes (DEGs) were screened, and a risk score comprising four miRNAs was constructed. We evaluated and tested the model accuracy through the ROC curve and a survival status analysis. Four-miRNA nomography were performed to quantitatively and visually evaluate the model. The potential biological functions of those network genes were performed through KEGG pathways and Gene Ontology (GO) terms analysis. Furthermore, survival analysis and the clinical information was used to verify whether the hub genes are associated with prognosis. The CGGA and GEPIA database were used to verified the accuracy of these analysis. To investigate the potential functions of those hub genes in glioma, we performed GSEA and GSVA.

Results The results of univariate Cox regression showed that twenty miRNAs were significantly associated with overall survival of glioma. Multivariate Cox regression analyses were performed to screen four miRNAs (hsa-miR-744-5p, hsa-miR-1249-3p, hsa-miR-3677-3p and hsa-miR-10b-3p). The estimated area under the receiver operating characteristic (ROC) curve (AUC) were 0.902, 0.896 and 0.907 for groups train, test and entire, respectively, indicating that the model had a significant ability to predict survival in glioma patients. Furthermore, univariate and multivariate Cox regression were performed using relevant clinical factors associated with glioma, showed that the model could act as an independent prognostic factor. CGGA and GEPIA database were used to verified the accuracy of these hub genes. GSEA and GSVA revealed that the lower expressions of these hub genes were most closely associated with vascularization and tumor proliferation.

Conclusions In conclusion, the four-miRNA risk score model and these hub genes may represent a novel prognostic marker to predict the survival of patients with glioma.

\section{Background}

Glioma is one of the most aggressive brain tumor that constitute approximately $80 \%$ of primary malignant tumors [1]. According to the degree of aggressive and malignant behavior by the World Health Organization (WHO), gliomas traditionally have been divided into four groups, denoted as I to IV, which describes them in terms of histopathologic lineage as either oligodendroglioma, astrocytoma, ependymoma, or mixed tumors [2]. Extensive progress has been achieved regarding to the early diagnosis, but the majority of glioma diagnoses are made at the advanced stage. Although significant improvements have been carried out on the treatment of glioma, including surgery, chemotherapy and radiotherapy, the overall survival rates for patients continue to be dismal. Therefore, it is important to develop new reliable diagnostic and prognostic biomarkers for glioma.

MicroRNAs (miRNAs) are a group of endogenous, single-stranded non-coding RNAs of approximately 18-24 nucleotides that play crucial regulatory roles in gene expression through interacting with the $3^{\prime}$-untranslated regions (3'-UTRs) of target mRNAs, leading to subsequent translational repression [3]. MiRNAs are essential for maintaining homeostasis in health and disease by finely tuning the expression of genes [4, 5]. Low expression or overexpression of some miRNAs (tumour suppressors or oncogenic) also cause disruption in delicate equilibrium which are involved in several aggressive cancers [6]. Accumulating evidence suggests that various miRNAs contribute to cancer initiation, progression, metastasis, diagnosis, prognosis and have potential applications in cancer therapy in numerous malignancies [7, 8]. Several miRNAs are identified as being closely associated with cancers by binding oncogenes or tumour suppresser genes in tumour cells [9]. A growing body of research has suggested that some miRNAs are related to the oncogenesis, development and prognosis of glioma [10,11]. MiR-34a-5p has been found to inhibit glioma cell migration, proliferation and potentiate temozolomide-induced cytotoxicity by targeting high mobility group AT-hook 2 (HMGA2) [12]. It has been reported that miR-520e inhibits Wnt/ $\beta$-catenin signaling by blinding to fibroblast growth factor 19 during glioma cells invasion and proliferation [13]. MiR-195, miR-15b and miR-103 have been shown to be tumors suppressors in glioma cells by downregulating SALL4 expression and suppressing glioma cell proliferation and invasion [14]. Therefore, studying the role of miRNA involved in the initiation and progression of gliomas is essential for making miRNA therapy for gliomas, and screening miRNA target genes for tumors is of vital importance during tumour research. Numerous miRNA-related bioinformatics technologies, such as microarray and RNA sequencing, chip sequencing, computational prediction, have been developed to validate miRNA targets, and miRNA-target interaction databases have been established.

In this study, we constructed a four-miRNA risk score model that could effectively predict the survival prognosis of glioma patients. Analysing the functional pathways and biological processes affected by these miRNAs and screening the targeted hub genes may help to elucidate the molecular mechanism of glioma and identify therapeutic targets.

\section{Materials And Methods}

\section{Data acquisition and processing}

The miRNA, mRNA expression profiles and relevant clinical information were extracted from The Cancer Genome Atlas (TCGA) website (https://www.cancer.gov). The clinical information of hub genes was downloaded from the CGGA database (Chinese Glioma Genome Atlas) which we 


\section{Identification Of Differently Expressed Mirnas And Mrnas}

EdgeR package of $\mathrm{R}$ language 3.6.1 was used to screen differently expressed miRNAs (DEMs) and differently expressed mRNAs/genes (DEGs) with $|\log 2 \mathrm{FC}|>1$ and corrected $p$-values $(\mathrm{FDR})<0.05$. We selected patients who had a survival time $\geq 30$ days and conducted an analysis of mRNA integrated with miRNA expression profiles.

\section{Establishment Of A Prognostic Mirna Risk Score Model}

The R software "caret" package was used to classify patients into two groups (the train group and the test group). Univariate and multivariate Cox regression analyses were performed to establish the prognostic miRNA risk score model through the "Coxph" function and R language "direction = both" survival package [15]. By calculating the sum of each miRNA multiplied by its coefficient, a risk score comprising four miRNAs was constructed that was capable of predicting the prognosis of glioma. Depending on the median of the risk score, the patients were randomized to two groups: low-risk group and high-risk group. We evaluated and tested the model accuracy in the three groups: test group, training group and entire group. The accuracy of a prediction model for glioma using the receiver operating characteristic (ROC) curve has been undertaken by using "survival ROC" package [16]. A survival status analysis and four-miRNA nomography were performed to quantitatively and visually evaluate the model using the R language "survival" and "rms" packages. Subsequently, univariate and multivariate Cox regression analysis were performed to evaluate the independent prognostic risk factor between the risk score and other clinical parameters including age and gender of patients, and stage of disease. The validity of the risk score and clinical variables were further verified by using ROC curves.

\section{Network Between Mirnas And Degs And Their Functions}

Data analysis using the largest and most recognized resources (Target Scan, miRTarBase and miRDB) to reveal overlapping target by Perl language. The associations between the miRNA and the target genes of microRNA was visualized using Cytoscape 3.6.1. The potential biological functions of those network genes were analysed using the "cluster Profiler" and "org.Hs.eg.db" R packages for Kyoto Encyclopedia of Genes and Genomes (KEGG) database pathways and Gene Ontology (GO) terms. An adjusted $p$-value $<0.05$ and $q$ value $<0.05$ were considered to be significant [17].

\section{Identification Of Hub Genes Related To Prognostic Survival}

A protein-protein interaction (PPI) network of the targeted genes was obtained from the STRING database (https://string-db.org/) [18], parameter confidence was set to 0.400 . Subsequently, we identified the top 8 hub genes by using CytoHubba plug-in of Cytoscape 3.6.1. Furthermore, KaplanMeier survival analysis was used to verify whether the hub genes are associated with prognostic survival (log-rank test, $p$-values $<0.05$ ).

\section{Hub Genes Verification And Analysis}

CGGA and GEPIA database were used to verified the accuracy of these hub genes. The clinical information of cancer histology (Fig. 8), grade (Fig. 9A) and the isocitrate dehydrogenase (IDH) mutation status (Fig. 9B) were significant correlation with these hub genes expression. GEPIA database analysis indicated that those hub genes were significantly lower expression in cancer tissues, however, the MAPK1 was not significant expression between the normal and cancer tissues (Fig. 10). The survival prognosis analysis result of CGGA database showed that the higher these hub genes expression, the better prognosis patients had (Fig. 11). Therefore, those genes may play important role in the glioma development. Combined with all these analyses supposed that these gene were tumor suppressor genes.

\section{Statistical analysis}

R version 3.6.1 was used for statistical analyses, and a $p$-value $<0.05$ was considered to be significant $\left({ }^{\star} P<0.05,{ }^{\star \star} P<0.01\right)$.

\section{Results}

\section{Identification of DEMs and DEGs}

There were 267 differently expressed DEMs, including 134 upregulated DEMs and 133 downregulated DEMs. We also screened 1601 DEGs, 759 of which were upregulated and 842 of which were downregulated, through the TCGA database.

\section{Grouping of samples and construction of a prognostic miRNA risk score model}


We screened the entire group of patients $(\mathrm{N}=478)$ who had more than 30 days of follow-up time and combined it with clinical information. Then, the entire group was randomly classified into two groups as train group $(\mathrm{N}=240)$ and test group $(\mathrm{N}=238)$. The survival random forest and univariate Cox regression results showed that twenty miRNAs were established to be significantly correlated with overall survival in the training group with $p$-value $<$ 0.05 (Table 1). Ten miRNAs were subsequently performed for further analysis according to the multivariate Cox regression analysis. Moreover, KaplanMeier analysis revealed that these miRNAs were associated with the overall survival time of patients with $p$-value $<0.05$ (Fig. 2 ). Nevertheless, there were contradictions between prognosis survival analysis and these miRNAs exhibited expression in tumors, such as hsa-miR-10b-5p, hsa-miR-21-3p, hsa-miR-135a-3p, hsa-miR-181c-5p, hsa-miR-221-3p and hsa-miR-548ba. Finally, four miRNAs (hsa-miR-744-5p, hsa-miR-1249-3p, hsa-miR-3677-3p and hsa-miR-10b-3p) were selected by using multivariate Cox regression analysis (Table 1). A prognostic model was built based on calculating the sum of each miRNA multiplied by its corresponding coefficient: miRNA risk score $=(0.57 \times$ expression of hsa-miR-3677-3p $)+(0.66 \times$ expression of hsa-miR$10 b-3 p)$ - (0.52 x expression of hsa-miR-1249-3p) - (0.79 x expression of hsa-miR-744-5p).

\section{Verified the validity and quantitative of the miRNA risk score model}

Patients were randomized to two groups: high-risk group and low-risk group. Analysis of the Kaplan-Meier survival curves indicated that the overall survival was significantly higher in the low-risk group compared with that of the high-risk group in the test group $(p=7.435 \mathrm{E}-04)$, entire group $(p=$ $1.558 \mathrm{E}-10)$ and train group $(p=8.603 \mathrm{E}-09$; Fig. $3 \mathrm{~A}-\mathrm{C})$. The test group and entire group were tested, and the accuracy and effectiveness of the fourmiRNA risk score model were verified. The estimated area under the receiver operating characteristic (ROC) curve (AUC) of the test group, entire group and train group were $0.896,0.907$ and 0.902 , respectively, which showed that the prediction model has a better predictive ability (Fig. 3D-F). Then, we

built a survival status analysis for brief quantitative and visual analysis of the model (Fig. $3 \mathrm{G}-\mathrm{I}$ ). In addition, univariate and multivariate Cox regression were performed using relevant clinical factors associated with glioma, showed that the four-miRNA risk score model could act as an independent prognostic factor (Table 2). Univariate Cox regression analysis showed that the risk score was markedly related to overall survival in patients (hazard ratio $\mathrm{HR}=1.010$, confidence interval $95 \% \mathrm{Cl}=1.007-1.013, p<0.01$ ). Multivariate Cox regression analysis revealed that the risk score was independent factor of overall survival, considering other clinical factors (hazard ratio HR $=1.008$, confidence interval $95 \% \mathrm{Cl}=1.004-1.012, p<0.01$; Table 2 ), such as clinical grade and age. The multivariate ROC curve indicated that risk score (0.908) had a high predictive ability (Fig. 4A). Finally, we built a nomography for quantitative and visual analysis of the four-miRNA risk score model (Fig. 4B). 
Table 1

Univariate and multivariate Cox regression of differentially expressed miRNAs.

\begin{tabular}{|c|c|c|c|c|c|c|c|c|c|}
\hline \multirow[t]{2}{*}{ ID } & \multicolumn{4}{|c|}{ Univariate Cox regression } & \multicolumn{5}{|c|}{ Multivariate Cox regression } \\
\hline & HR & HR.95L & HR.95H & $P$ value & Co ef & HR & HR.95L & HR.95H & $P$ value \\
\hline hsa-miR-10b-3p & 1.228 & 1.103 & 1.367 & 0.000 & 0.664 & 1.942 & 1.292 & 2.917 & 0.001 \\
\hline hsa-miR-1249-3p & 0.567 & 0.446 & 0.721 & 0.000 & 0.518 & 0.596 & 0.409 & 0.867 & 0.007 \\
\hline hsa-miR-3677-3p & 1.316 & 1.075 & 1.612 & 0.008 & 0.570 & 1.768 & 1.295 & 2.415 & 0.000 \\
\hline hsa-miR-744-5p & 0.542 & 0.407 & 0.723 & 0.000 & 0.795 & 0.452 & 0.283 & 0.720 & 0.001 \\
\hline hsa-miR-128-3p & 0.582 & 0.462 & 0.732 & 0.000 & & & & & \\
\hline hsa-miR-1343-3p & 0.709 & 0.550 & 0.913 & 0.008 & & & & & \\
\hline hsa-miR-139-5p & 0.694 & 0.561 & 0.859 & 0.001 & & & & & \\
\hline hsa-miR-181a-2-3p & 0.711 & 0.558 & 0.907 & 0.006 & & & & & \\
\hline hsa-miR-21-5p & 1.264 & 1.075 & 1.486 & 0.005 & & & & & \\
\hline hsa-miR-222-3p & 1.389 & 1.098 & 1.757 & 0.006 & & & & & \\
\hline hsa-miR-3200-3p & 0.586 & 0.468 & 0.734 & 0.000 & & & & & \\
\hline hsa-miR-491-3p & 0.721 & 0.572 & 0.908 & 0.006 & & & & & \\
\hline hsa-miR-497-5p & 0.651 & 0.483 & 0.877 & 0.005 & & & & & \\
\hline hsa-miR-892a & 0.805 & 0.694 & 0.934 & 0.004 & & & & & \\
\hline hsa-miR-10b-5p & 1.148 & 1.047 & 1.260 & 0.003 & & & & & \\
\hline hsa-miR-135a-3p & 0.790 & 0.677 & 0.921 & 0.003 & & & & & \\
\hline hsa-miR-181c-5p & 0.645 & 0.500 & 0.831 & 0.001 & & & & & \\
\hline hsa-miR-21-3p & 1.301 & 1.127 & 1.500 & 0.000 & & & & & \\
\hline hsa-miR-221-3p & 1.424 & 1.122 & 1.807 & 0.004 & & & & & \\
\hline hsa-miR-548ba & 0.721 & 0.600 & 0.865 & 0.000 & & & & & \\
\hline
\end{tabular}

\section{Network Between Mirnas And Degs And Functional Enrichment Analysis}

There were 195 overlapping genes identified between miRNA target genes and DEGs. The network was built on those genes (Fig. 5A) and screened the eight hub genes (Fig. 5B). We predicted the potential biological functions of those genes through KEGG signaling pathway and GO enrichment analysis. The GO and KEGG pathway analysis results are listed in Tables 3 and 4, respectively. A $p$-value $<0.05$ was used as a cut-off point. The top terms of GO annotation in biological process (BP), molecular function (MF) and cellular component (CC) are displayed. The enriched GO functions for target genes of the miRNAs were primarily associated with neural axonogenesis and synapses (Fig. 6A-F). The KEGG pathway analysis indicated that cancer-related pathways were clearly activated, including the cAMP signaling pathway, oocyte meiosis pathway and MAPK signaling pathway

(Fig. 6G-I).

Table 2

Univariate and multivariate Cox regression of clinical features.

\begin{tabular}{|lllllllll|}
\hline Clinical features & \multicolumn{3}{l}{ Univariate Cox regression } & \multicolumn{5}{c|}{ Multivariate Cox regression } \\
\cline { 2 - 9 } & HR & HR.95L & HR.95H & P value & HR & HR.95L & HR.95H & P value \\
\hline Age & 1.058 & 1.043 & 1.074 & 0.000 & 1.055 & 1.039 & 1.071 & 0.000 \\
\hline Gender & 1.063 & 0.742 & 1.522 & 0.739 & 1.146 & 0.788 & 1.668 & 0.476 \\
\hline Grade & 3.313 & 2.229 & 4.926 & 0.000 & 2.694 & 1.792 & 4.051 & 0.000 \\
\hline Risk Score & 1.010 & 1.007 & 1.013 & 0.000 & 1.008 & 1.004 & 1.012 & 0.000 \\
\hline GO analysis of target genes associated glioma. & & & & & \\
\hline
\end{tabular}


GO analysis of target genes associated glioma.

\begin{tabular}{|c|c|c|c|c|}
\hline ID & Description & $P$ adjust & $Q$ value & Count \\
\hline \multicolumn{5}{|l|}{ BP } \\
\hline G0:0007409 & axonogenesis & 6.06E-09 & 4.95E-09 & 25 \\
\hline G0:0050804 & modulation of chemical synaptic transmission & 6.06E-09 & 4.95E-09 & 24 \\
\hline G0:0099177 & regulation of trans-synaptic signaling & $6.06 \mathrm{E}-09$ & 4.95E-09 & 24 \\
\hline G0:0016358 & dendrite development & 8.36E-08 & 6.83E-08 & 17 \\
\hline G0:0048167 & regulation of synaptic plasticity & $2.31 \mathrm{E}-07$ & 1.89E-07 & 15 \\
\hline G0:0010975 & regulation of neuron projection development & $2.76 \mathrm{E}-07$ & $2.26 \mathrm{E}-07$ & 23 \\
\hline G0:0050890 & cognition & 1.87E-06 & $1.53 \mathrm{E}-06$ & 17 \\
\hline G0:0010769 & regulation of cell morphogenesis involved in differentiation & $2.11 \mathrm{E}-06$ & $1.72 \mathrm{E}-06$ & 17 \\
\hline G0:0007611 & learning or memory & $9.35 \mathrm{E}-06$ & 7.65E-06 & 15 \\
\hline G0:0022604 & regulation of cell morphogenesis & 1.45E-05 & 1.18E-05 & 20 \\
\hline \multicolumn{5}{|l|}{$\mathrm{CC}$} \\
\hline G0:0098984 & neuron to neuron synapse & $6.68 \mathrm{E}-09$ & 4.97E-09 & 21 \\
\hline G0:0014069 & postsynaptic density & 3.86E-08 & 2.87E-08 & 19 \\
\hline G0:0032279 & asymmetric synapse & 3.86E-08 & 2.87E-08 & 19 \\
\hline G0:0099572 & postsynaptic specialization & 7.81E-08 & 5.80E-08 & 19 \\
\hline G0:0097060 & synaptic membrane & 3.77E-07 & 2.81E-07 & 20 \\
\hline G0:0098978 & glutamatergic synapse & 1.29E-05 & $9.62 \mathrm{E}-06$ & 16 \\
\hline G0:0043025 & neuronal cell body & 1.33E-05 & $9.90 \mathrm{E}-06$ & 19 \\
\hline G0:0098793 & presynapse & 4.46E-05 & 3.31E-05 & 18 \\
\hline G0:0033267 & axon part & 0.000141983 & 0.000105525 & 15 \\
\hline G0:0045211 & postsynaptic membrane & 0.00041505 & 0.000308474 & 13 \\
\hline \multicolumn{5}{|l|}{ MF } \\
\hline G0:0004674 & protein serine/threonine kinase activity & 0.000937207 & 0.000819795 & 17 \\
\hline G0:0004712 & protein serine/threonine/tyrosine kinase activity & 0.000937207 & 0.000819795 & 6 \\
\hline G0:0004707 & MAP kinase activity & 0.001329149 & 0.001162636 & 4 \\
\hline GO:0004708 & MAP kinase kinase activity & 0.001783992 & 0.001560497 & 4 \\
\hline G0:0016247 & channel regulator activity & 0.009026849 & 0.007895983 & 8 \\
\hline GO:0017075 & syntaxin-1 binding & 0.009026849 & 0.007895983 & 4 \\
\hline G0:0015631 & tubulin binding & 0.01001915 & 0.008763971 & 12 \\
\hline G0:0099106 & ion channel regulator activity & 0.010524542 & 0.009206049 & 7 \\
\hline G0:0008179 & adenylate cyclase binding & 0.011666652 & 0.010205077 & 3 \\
\hline G0:0030276 & clathrin binding & 0.011666652 & 0.010205077 & 5 \\
\hline
\end{tabular}

\section{Survival Prognosis Analysis Of The Hub Genes}

According the node degrees, eight hub genes were finally screened out by the cytoHubba plug-in, including SNAP25, MAPK1, CAMK2A, SYT1, NRXN1, CALM1, ATP2B2, and CALM3 in Table 5. Kaplan-Meier survival analysis indicated that the eight top hub genes were positively associated with the survival prognosis of glioma (Fig. 7). 
Table 4

KEGG pathways of target genes associated glioma.

\begin{tabular}{|lllll|}
\hline ID & Description & Padjust & Q value & Count \\
\hline hsa04024 & cAMP signaling pathway & $1.13 \mathrm{E}-06$ & $6.36 \mathrm{E}-07$ & 15 \\
\hline hsa04114 & Oocyte meiosis & $8.27 \mathrm{E}-06$ & $4.65 \mathrm{E}-06$ & 11 \\
\hline hsa04720 & Long-term potentiation & $2.43 \mathrm{E}-05$ & $1.36 \mathrm{E}-05$ & 8 \\
\hline hsa04912 & GnRH signaling pathway & $2.43 \mathrm{E}-05$ & $1.36 \mathrm{E}-05$ & 9 \\
\hline hsa04022 & cGMP-PKG signaling pathway & $4.91 \mathrm{E}-05$ & $2.76 \mathrm{E}-05$ & 11 \\
\hline hsa04722 & Neurotrophin signaling pathway & 0.000130086 & $7.32 \mathrm{E}-05$ & 9 \\
\hline hsa04925 & Aldosterone synthesis and secretion & 0.000224145 & 0.000126086 & 8 \\
\hline hsa05214 & Glioma & 0.000307502 & 0.000172975 & 7 \\
\hline hsa04360 & Axon guidance & 0.000407607 & 0.000229286 & 10 \\
\hline hsa04012 & ErbB signaling pathway & 0.000532368 & 0.000299466 & 7 \\
\hline Identification of hub genes by cytoHubba & & & \\
\hline
\end{tabular}

Table 5

Identification of hub genes by cytoHubba

\begin{tabular}{|lllllllllllll|}
\hline Node_name & MCC & DMNC & MNC & Degree & EPC & $\begin{array}{l}\text { Bottle } \\
\text { Neck }\end{array}$ & $\begin{array}{l}\text { EC } \\
\text { centricity }\end{array}$ & Closeness & Radiality & $\begin{array}{c}\text { Betweenness } \\
\text { Coefficient }\end{array}$ \\
\hline SNAP25 & 499 & 0.307 & 20 & 25 & 47.235 & 18 & 0.182 & 67.267 & 6.970 & 3413.216 & 12218 & 0.167 \\
\hline MAPK1 & 121 & 0.174 & 23 & 24 & 46.861 & 26 & 0.152 & 65.450 & 6.868 & 2787.012 & 10830 & 0.130 \\
\hline CAMK2A & 286 & 0.266 & 21 & 21 & 47.712 & 15 & 0.182 & 67.200 & 7.039 & 2581.640 & 11404 & 0.224 \\
\hline NRXN1 & 75 & 0.293 & 12 & 15 & 43.184 & 7 & 0.182 & 60.817 & 6.854 & 2033.366 & 6548 & 0.190 \\
\hline SYT1 & 448 & 0.391 & 15 & 15 & 46.261 & 9 & 0.182 & 60.417 & 6.806 & 804.941 & 4348 & 0.371 \\
\hline ATP2B2 & 272 & 0.468 & 12 & 14 & 45.866 & 10 & 0.182 & 58.367 & 6.717 & 614.824 & 3080 & 0.352 \\
\hline CALM1 & 222 & 0.338 & 14 & 14 & 45.631 & 7 & 0.152 & 61.500 & 6.861 & 1062.399 & 5610 & 0.330 \\
\hline SYT4 & 388 & 0.492 & 11 & 13 & 43.439 & 6 & 0.152 & 55.767 & 6.566 & 438.769 & 1798 & 0.385 \\
\hline
\end{tabular}

\section{Potential Functions Of These Hub Genes}

To further investigate the potential functions of those hub genes in glioma, we performed GSEA and GSVA. As shown in Fig. 12, the high expression of these hub genes were most enriched in "long term potentiation", "neuroactive ligand receptor interaction" and "phosphatidylinositol signaling system" pathways. These pathways were closely associated with signal transduction and synaptic repair. The lower expressions of these hub genes were most enriched in "p53 signaling pathway", "antigen processing and presentation" and "complement and coagulation cascades" pathways. These gene sets with the highest enrichment scores were most closely associated with vascularization and tumor proliferation. Furthermore, GSVA verified that these gene sets were significantly upregulated in the lower expression (Fig. 13).

\section{Discussion}

Glioma is a highly malignant brain tumour that has a high risk of recurrence and mortality, and a poor prognosis [19]. Thus, identifying more sensitive, specific and timely biomarkers for diagnosis, as well as investigating the mechanisms of development and progression in glioma, is becoming increasingly urgent [20]. A growing body of research has demonstrated that distinct miRNA expression profiles have been identified as a crucial factor responsible for the initiation and progression of various human malignant cancers [21,22]. The differential expression of miRNA targeted genes in tumor tissues may be triggered by several factors, including positional effects of cancer-related genome regions, epigenetic regulatory mechanisms, and alterations in the miRNA processing machinery [23]. MiRNAs are involved in tumor invasion by targeting many critical protein-coding genes [24]. Recent studies have highlighted that miRNAs play important roles in cancer development, indicating that miRNAs have strong potential to become clinically useful diagnostic markers for early cancer detection [25, 26]. The multiple miRNA risk score is superior to a single miRNA in terms of statistically robust analysis. Although many studies have reported many prognostic markers implicated in glioma prognosis at present, using miRNA risk scores and sample groupings to validate the model has not been supported by previous studies. 
In this study, we built a four-miRNA risk score model (i.e., hsa-miR-744-5p, hsa-miR-1249-3p, hsa-miR-3677-3p, and hsa-miR-10b-3p) that effectively predicted patient survival. Accumulating evidence suggests that most of these four miRNAs are participated in various bioactivities during tumorigenesis. M. Kleemann et al. reported that miR-744-5p expression was significantly decreased in cancer cell lines, and the miR-744-5p-mediated apoptosis signaling pathway revealed that the overexpression of miR-744-5p directly inhibited heterogeneous nuclear ribonucleoprotein C (HNRNPC) expression and nuclear factor I X (NFIX) [27]. H. Shu et al. showed that the miR-1249-3p was increased in hepatocellular carcinoma (HCC) cell lines, functioning as an oncogene in cell proliferation, migration, and cell invasion through targeting heterogeneous nuclear ribonucleoprotein $\mathrm{K}$, and high expression of miR-1249-3p was correlated with poor overall poor overall survival in patients with HCC [28]. Zhang Q et al. demonstrated that miR-10b$3 p$ regulated cell proliferation, apoptosis, migration, and invasion of oesophageal cancer cells (ESCC) under hypoxia, and the miR-10b-3p expression was significantly increased after hypoxia combined with hypoxia inducible factor 1a [29].

Meanwhile, the network between these miRNA target genes and the DEGs was described, functional analysis and pathway enrichment analysis were performed on the network genes. GO enrichment analysis illustrated that those target genes were primarily participated in the regulation of cell morphogenesis and neuron projection development, modulation of chemical synaptic transmission, synaptic membrane, glutamatergic synapse, neuron to neuron synapse, actin binding, protein serine/threonine kinase activity and channel regulator activity. The enriched pathways of target genes of included cAMP signalling pathway, oocyte meiosis pathway and long-term potentiation pathway and so on. All of these studies showed that these target genes played active role in promoting tumour maintenance to some degree. Thus, the four miRNAs have great significance in the regulation of tumour signaling pathways.

Finally, we screened eight hub genes (i.e., SNAP25, MAPK1, CAMK2A, SYT1, NRXN1, CALM1, ATP2B2, and CALM3). The result of Kaplan-Meier indicated that high expression of SNAP25, MAPK1, CAMK2A, SYT1, NRXN1, CALM1, ATP2B2, and CALM3 was associated with favourable prognosis in glioma patients. Therefore, we supposed that these genes were tumor suppressor genes. To further validated the relevance of our hub genes to clinical outcomes, we performed the CGGA and GEPIA database. In this study, the result showed that the glioma histology was significant correlation with these hub genes expression. It was well known that the higher the tumor grade, the worse the survival prognosis. Those hub genes were lower expression in higher grade glioma. Many studies had reported that IDH mutant gliomas had better overall survival [30-32]. In this study, these hub genes were also higher expression in IDH mutant glioma. The GEPIA database analysis indicated that those hub genes were significantly lower expression in cancer tissues. The survival prognosis analysis result of CGGA database also showed that the higher these hub genes expression, the better prognosis patients had. GSEA and GSVA also revealed that the lower expressions of these hub genes were most closely associated with vascularization and tumor proliferation. These results further verified our hypothesis and illustrated the reliability of our research that these hub genes might play more important roles in the glioma development and act as tumor suppressor genes. Synaptosomal-associated protein 25 (SNAP25), a key hub gene in the PPI network that could regulate calcium dynamics and neuronal plasticity, was related to the favorable survival of patients. Many studies have reported that lower expression of SNAP25 contributes to the occurrence and progression of tumours, and the expression of SNAP25 involves in the restoration of dendrites which may lead to terminal differentiation with subsequent loss of tumorigenicity [33]. Therefore, these miRNAs may affect the survival prognosis of glioma patients by regulating these hub genes.

\section{Conclusions}

We constructed a four-miRNA prognosis risk score model that had good reliability and strong predictive ability that could represent as a novel prognostic marker in glioma. Further we screened 8 hub genes which acted as the tumor suppressor genes which were regulated by the four miRNAs. The present study analysed the miRNA and target mRNA data of glioma through multiple tumor databases using a bioinformatic approach. To verify the effectiveness and feasibility of this proposed model, other databases and numbers of experiments should be employed in future studies, and the model may provide a promising therapeutic target for glioma treatment.

\section{Declarations}

Acknowledgements

Not applicable.

\section{Authors' contributions}

SS L and ZZ M wrote the manuscript. XY H, JJ W and DF D analyzed the data and created the images. YC L and JG X contributed to the modification of the manuscript. All authors read and approved the final manuscript.

\section{Funding}

This work was financially supported by the National Key R\&D Program of China (2018YFC2001600).

\section{Availability of data and material}

The datasets used and/or analyzed during the current study are available from the corresponding author on reasonable request. 


\section{Ethics approval and consent to participate}

Not applicable

\section{Consent for publication}

Not applicable.

\section{Competing interests}

The authors have no conflicts of interest to declare.

\section{Author details}

${ }^{1}$ Shanghai University of Traditional Chinese Medicine, School of Rehabilitation Science, No. 1200, Cailun Road, Shanghai 201203 , China. ${ }^{2}$ Center of Rehabilitation Medicine, Yueyang Hospital of Integrated Traditional Chinese and Western Medicine, Shanghai University of Traditional Chinese Medicine, Shanghai, China. ${ }^{3}$ Department of Trauma and Orthopedics, Yueyang Hospital of Integrated Traditional Chinese and Western Medicine, Shanghai University of Traditional Chinese Medicine, Shanghai, China. ${ }^{4}$ Rehabilitation Section, Spine Surgery Division of Department of Orthopedics, Tongji Hospital Affiliated to Tongji University School of Medicine, Shanghai, China.

\section{References}

1. Schwartzbaum JA, Fisher JL, Aldape KD, et al. Epidemiology and molecular pathology of glioma [J]. Nat Clin Pract Neurol. 2006;2(9):494-503. quiz 491 p following 516 .

2. Cuddapah VA, Robel S, Watkins S, et al. A neurocentric perspective on glioma invasion [J]. Nat Rev Neurosci. 2014;15(7):455-65.

3. Bartel DP. MicroRNAs: target recognition and regulatory functions [J]. Cell. 2009;136(2):215-33.

4. Li J, Lei L, Ye F, et al. Nutritive implications of dietary microRNAs: facts, controversies, and perspectives [J]. Food Funct. 2019;10(6):3044-56.

5. Bartel DP. MicroRNAs: genomics, biogenesis, mechanism, and function [J]. Cell. 2004;116(2):281-97.

6. Farazi TA, Spitzer JI, Morozov P, et al. miRNAs in human cancer [J]. J Pathol. 2011;223(2):102-15.

7. Babaei K, Shams S, Keymoradzadeh A, et al. An insight of microRNAs performance in carcinogenesis and tumorigenesis; an overview of cancer therapy [J]. Life Sci. 2020;240:117077.

8. Calin GA, Croce CM. MicroRNA signatures in human cancers [J]. Nat Rev Cancer. 2006;6(11):857-66.

9. Di Leva G, Garofalo M, Croce CM. MicroRNAs in cancer [J]. Annu Rev Pathol. 2014;9:287-314.

10. Zhou Q, Liu J, Quan J, et al. MicroRNAs as potential biomarkers for the diagnosis of glioma: A systematic review and meta-analysis [J]. Cancer Sci. 2018;109(9):2651-9.

11. Ye X, Wei W, Zhang Z, et al. Identification of microRNAs associated with glioma diagnosis and prognosis [J]. Oncotarget. 2017;8(16):26394-403.

12. Ma S, Fu T, Zhao S, et al. MicroRNA-34a-5p suppresses tumorigenesis and progression of glioma and potentiates Temozolomide-induced cytotoxicity for glioma cells by targeting HMGA2 [J]. Eur J Pharmacol. 2019;852:42-50.

13. Zhang L, Cao Y, Jia D, et al. MicroRNA-520e restricts the proliferation and invasion of glioma cells through the downregulation of Wnt/beta-catenin signaling by targeting fibroblast growth factor 19 [J]. Biochem Biophys Res Commun. 2019;511(3):619-25

14. Chen LP, Zhang NN, Ren XQ, et al. miR-103/miR-195/miR-15b Regulate SALL4 and Inhibit Proliferation and Migration in Glioma [J]. Molecules. 2018;23(11).

15. Stel VS, Dekker FW, Tripepi G, et al. Survival analysis II: Cox regression [J]. Nephron Clin Pract. 2011;119(3):c255-260.

16. Heagerty PJ, Lumley T, Pepe MS. Time-dependent ROC curves for censored survival data and a diagnostic marker [J]. Biometrics. 2000;56(2):33744.

17. Yang G, Zhang Y, Yang J. A Five-microRNA Signature as Prognostic Biomarker in Colorectal Cancer by Bioinformatics Analysis [J]. Front Oncol. 2019;9:1207.

18. Szklarczyk D, Morris JH, Cook H, et al. The STRING database in 2017: quality-controlled protein-protein association networks, made broadly accessible [J]. Nucleic Acids Res. 2017;45(D1):D362-8.

19. Ohgaki H. Epidemiology of brain tumors [J]. Methods Mol Biol. 2009;472:323-42.

20. Rupaimoole R, Slack FJ. MicroRNA therapeutics: towards a new era for the management of cancer and other diseases [J]. Nat Rev Drug Discov. 2017;16(3):203-22.

21. Schickel R, Boyerinas B, Park SM, et al. MicroRNAs: key players in the immune system, differentiation, tumorigenesis and cell death [J]. Oncogene. 2008;27(45):5959-74.

22. Mizoguchi M, Guan Y, Yoshimoto K, et al. MicroRNAs in Human Malignant Gliomas [J]. J Oncol. 2012;2012:732874.

23. Kunej T, Godnic I, Horvat S, et al. Cross talk between microRNA and coding cancer genes [J]. Cancer J. 2012;18(3):223-31.

Page 9/18 
24. Li X, Yu X, He Y, et al. Integrated Analysis of MicroRNA (miRNA) and mRNA Profiles Reveals Reduced Correlation between MicroRNA and Target Gene in Cancer [J]. Biomed Res Int. 2018;2018:1972606.

25. Wang C, Hu J, Lu M, et al. A panel of five serum miRNAs as a potential diagnostic tool for early-stage renal cell carcinoma [J]. Sci Rep. 2015;5:7610.

26. Ferracin M, Veronese A, Negrini M. Micromarkers: miRNAs in cancer diagnosis and prognosis [J]. Expert Rev Mol Diagn. 2010;10(3):297-308.

27. Kleemann M, Schneider H, Unger K, et al. MiR-744-5p inducing cell death by directly targeting HNRNPC and NFIX in ovarian cancer cells [J]. Sci Rep. 2018;8(1):9020.

28. Shu H, Hu J, Deng H. miR-1249-3p accelerates the malignancy phenotype of hepatocellular carcinoma by directly targeting HNRNPK [J]. Mol Genet Genomic Med. 2019;7(10):e00867.

29. Lu YF, Yu JR, Yang Z, et al. Promoter hypomethylation mediated upregulation of MicroRNA-10b-3p targets FOXO3 to promote the progression of esophageal squamous cell carcinoma (ESCC) [J]. J Exp Clin Cancer Res. 2018;37(1):301.

30. Eckel-Passow JE, Lachance DH, Molinaro AM, et al. Glioma Groups Based on 1p/19q, IDH, and TERT Promoter Mutations in Tumors [J]. N Engl J Med. 2015;372(26):2499-508.

31. Li G, Wang Z, Zhang C, et al. MEGF10, a Glioma Survival-Associated Molecular Signature, Predicts IDH Mutation Status [J]. Dis Markers. 2018;2018:5975216.

32. Malta TM, de Souza CF, Sabedot TS, et al. Glioma CpG island methylator phenotype (G-CIMP): biological and clinical implications [J]. Neuro Oncol. 2018;20(5):608-20.

33. Huang CJ, Lee CL, Liu CY, et al. Detection of lower levels of SNAP25 using multiple microarray systems and its functional significance in medulloblastoma [J]. Int J Mol Med. 2017;39(5):1195-205.

\section{Figures}

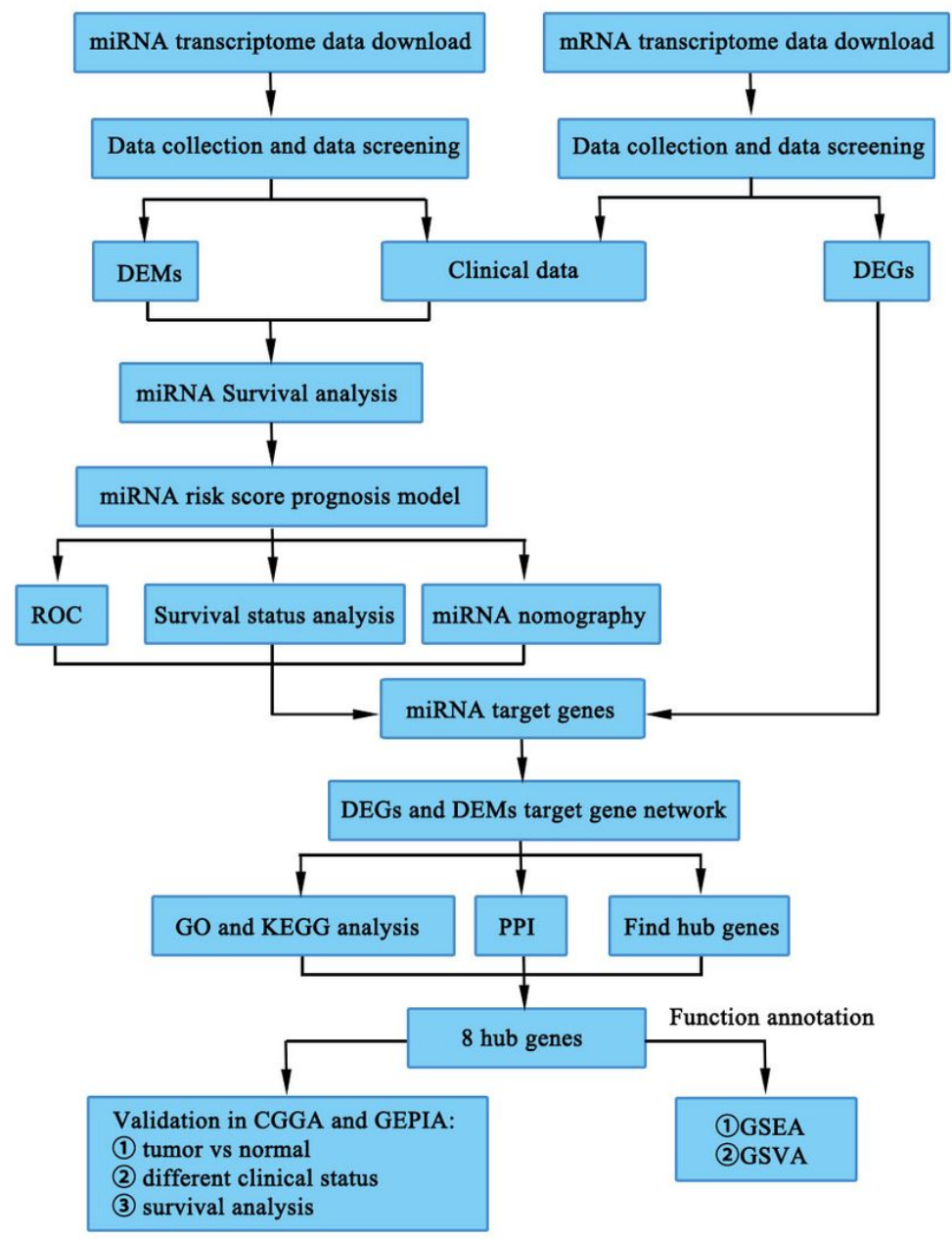

Figure 1 
Flowchart of the bioinformatic analysis.
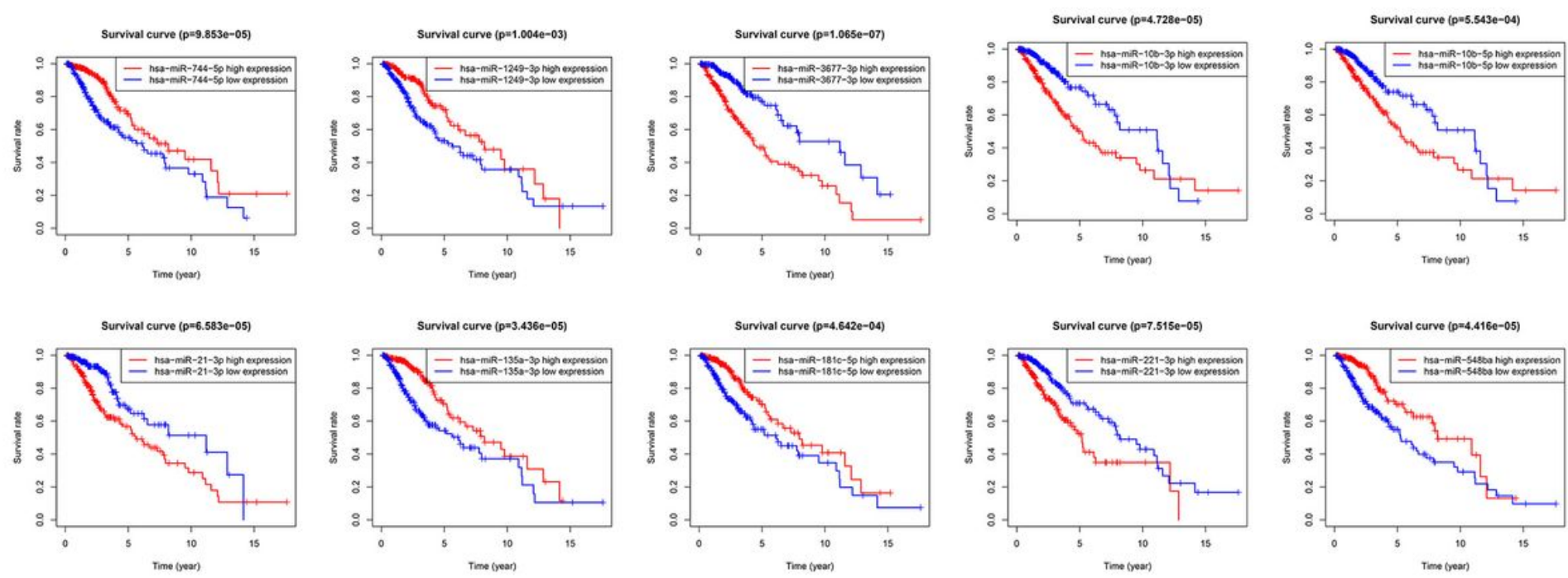

Figure 2

Ten miRNAs associated with overall survival in glioma patients.

A



D

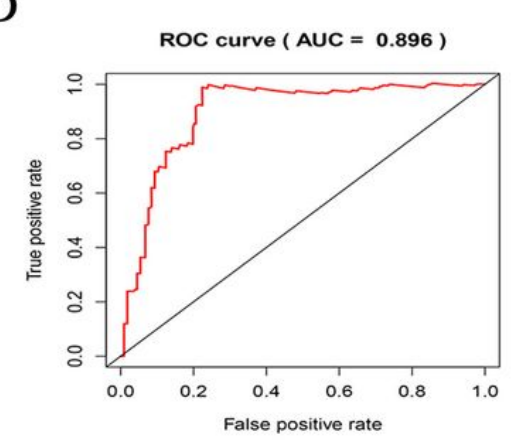

G

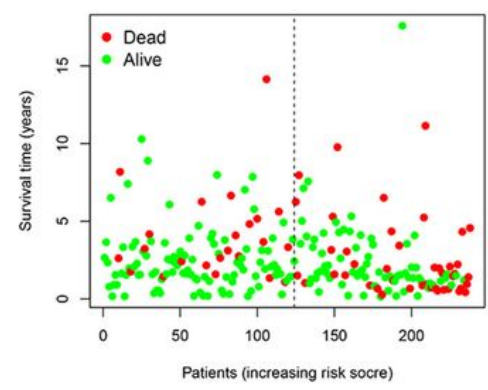

B

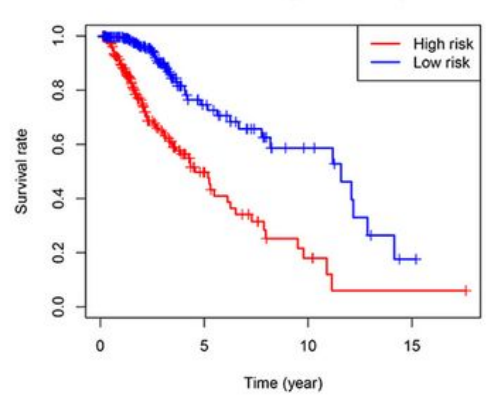

E



$\mathrm{H}$

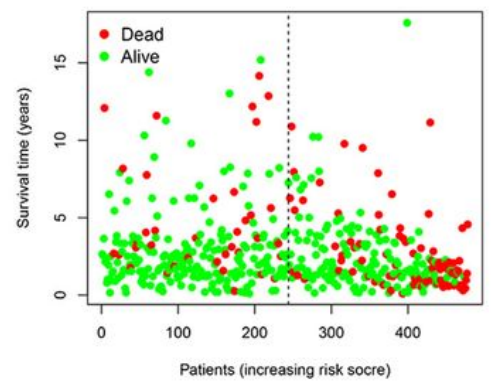

C

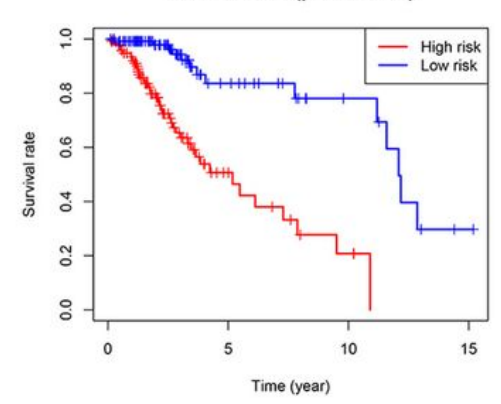

F

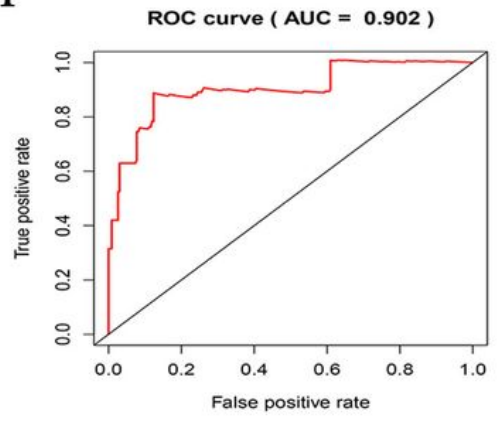

I

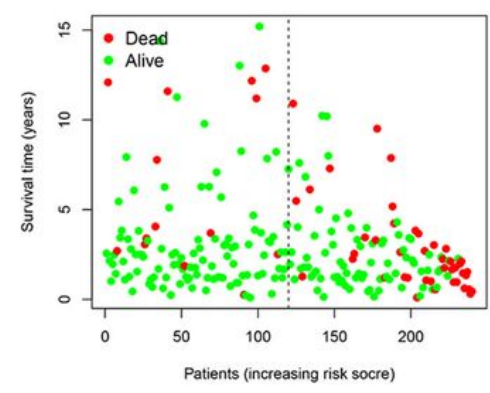

Figure 3 
Tested and verified prognosis of the four-miRNA risk score model. Kaplan-Meier curves in the training group (A), test group (B), and entire group (C). The AUC-dependent curve in the training group (D), test group (E), entire group (F), survival status in high- and low-risk patients for the training group $(G)$, test group $(H)$, and entire group $(I)$. Red dots represent death, and green dots represent survival.
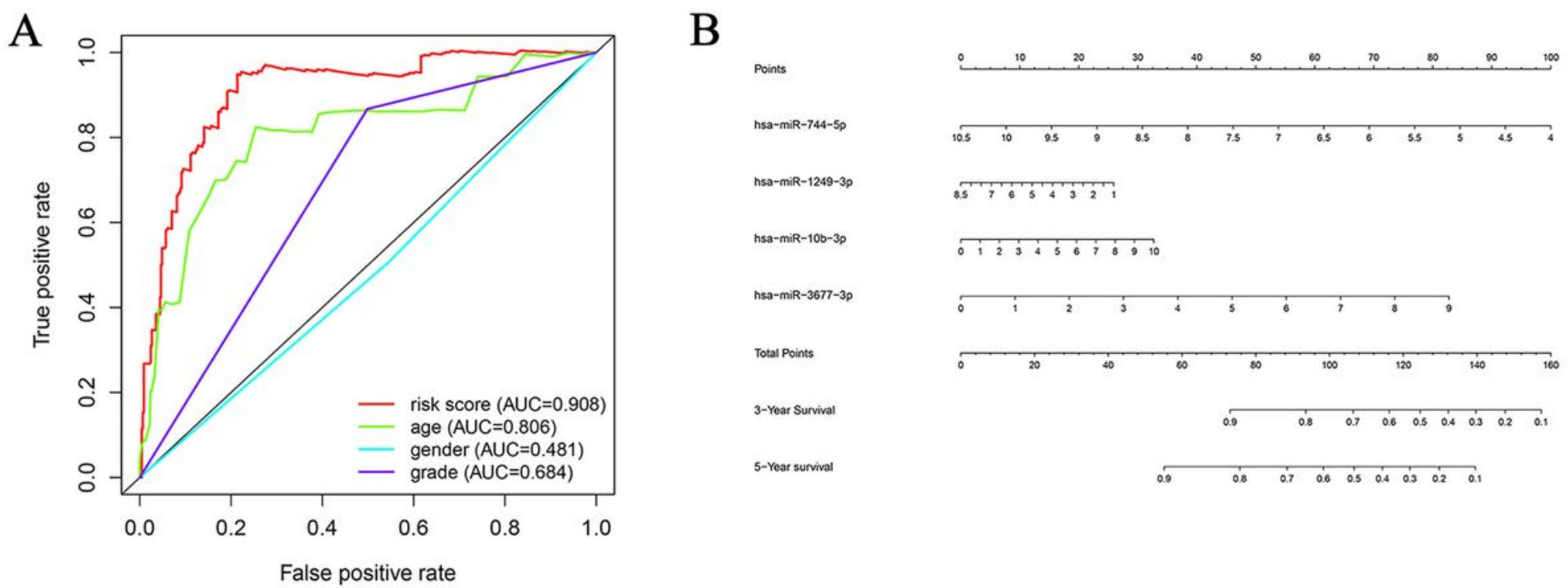

\section{Figure 4}

Verification and visualization of the miRNA risk score model. (A) The multivariate ROC curve demonstrated that risk score (0.908), age (0.806), and clinical grade (0.684) had a high predictive ability. (B) Nomography of the four-miRNA risk score model.

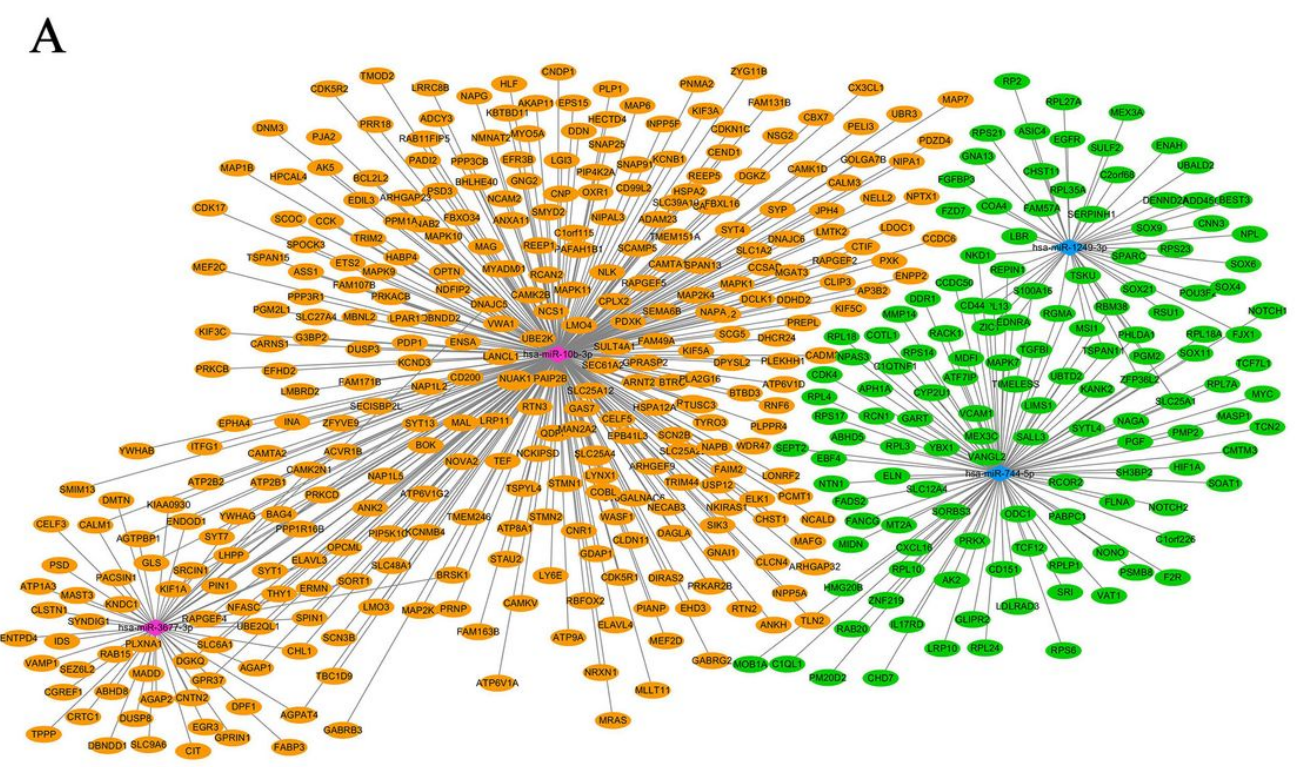

\section{B}

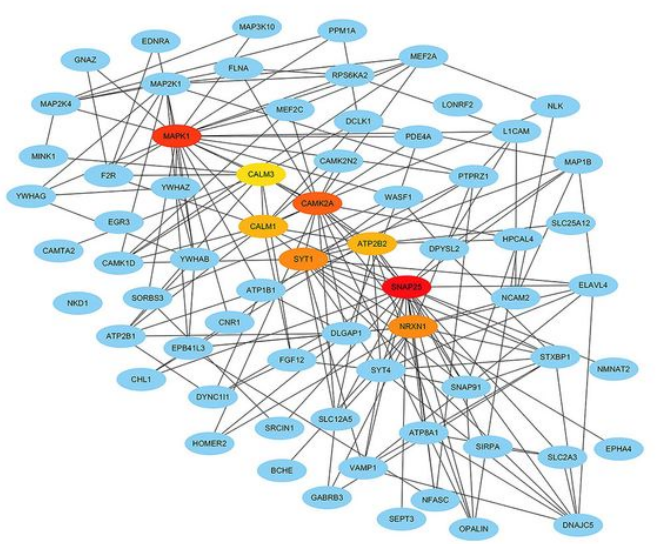




\section{Figure 5}

(A) Network map of miRNA regulating mRNA. Orange means upregulated genes, green means downregulated genes, pink means upregulated miRNAs, blue means downregulated miRNAs. (B) Hub genes of the PPI network. The redder the colour is, the larger the degrees are.

A

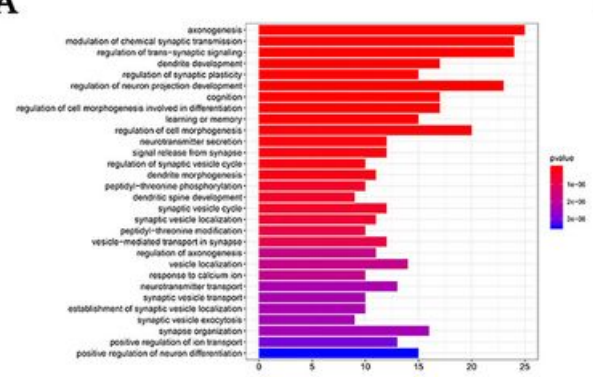

B

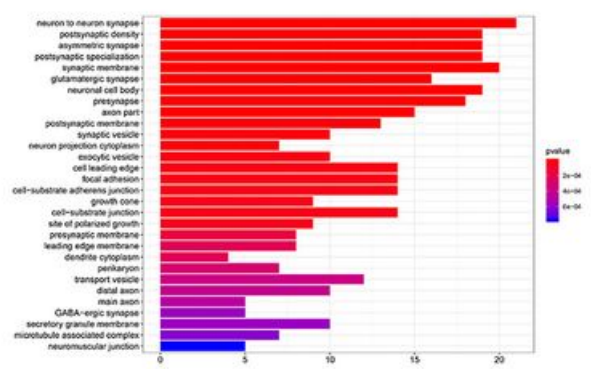

C

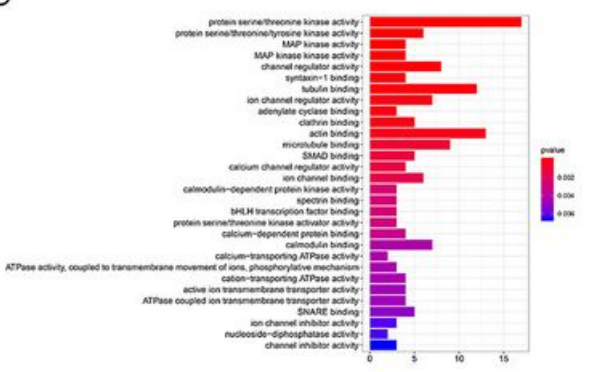

E
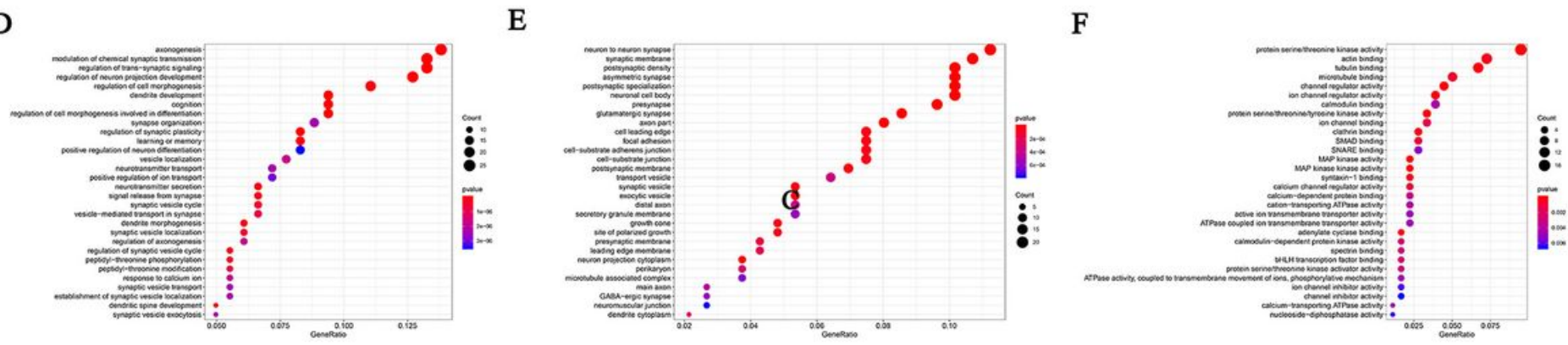

$\mathrm{H}$

I
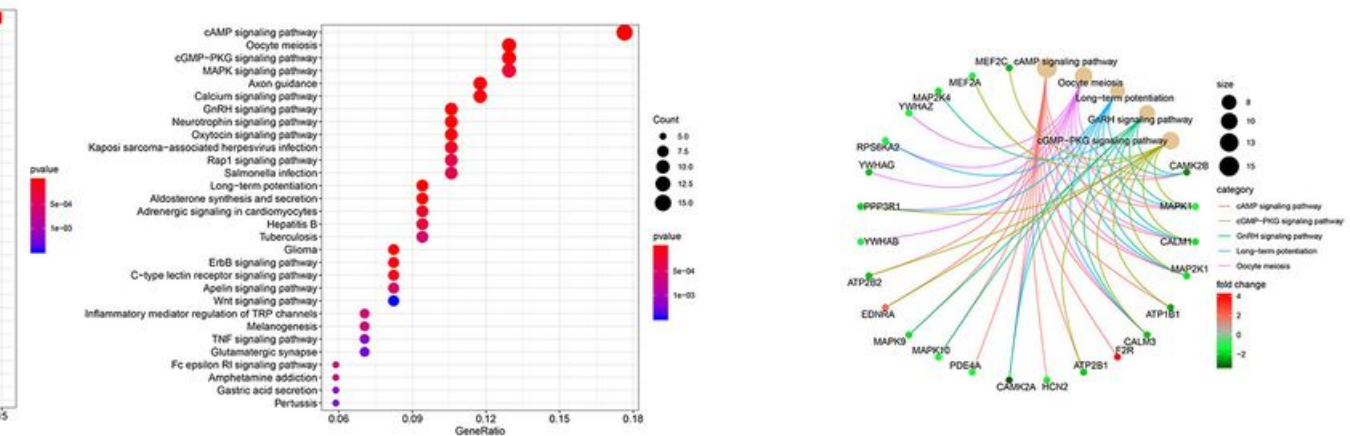

Figure 6

Functional enrichment analysis of network genes. (A, D) BP, (B, E) CC, (C, F) MF. (G, H) KEGG signal pathway analysis, and dot plot of KEGG signal pathway shown the counts of genes. (I) Cnetplot of KEGG signal pathway shown the "pathway-gene" network. 

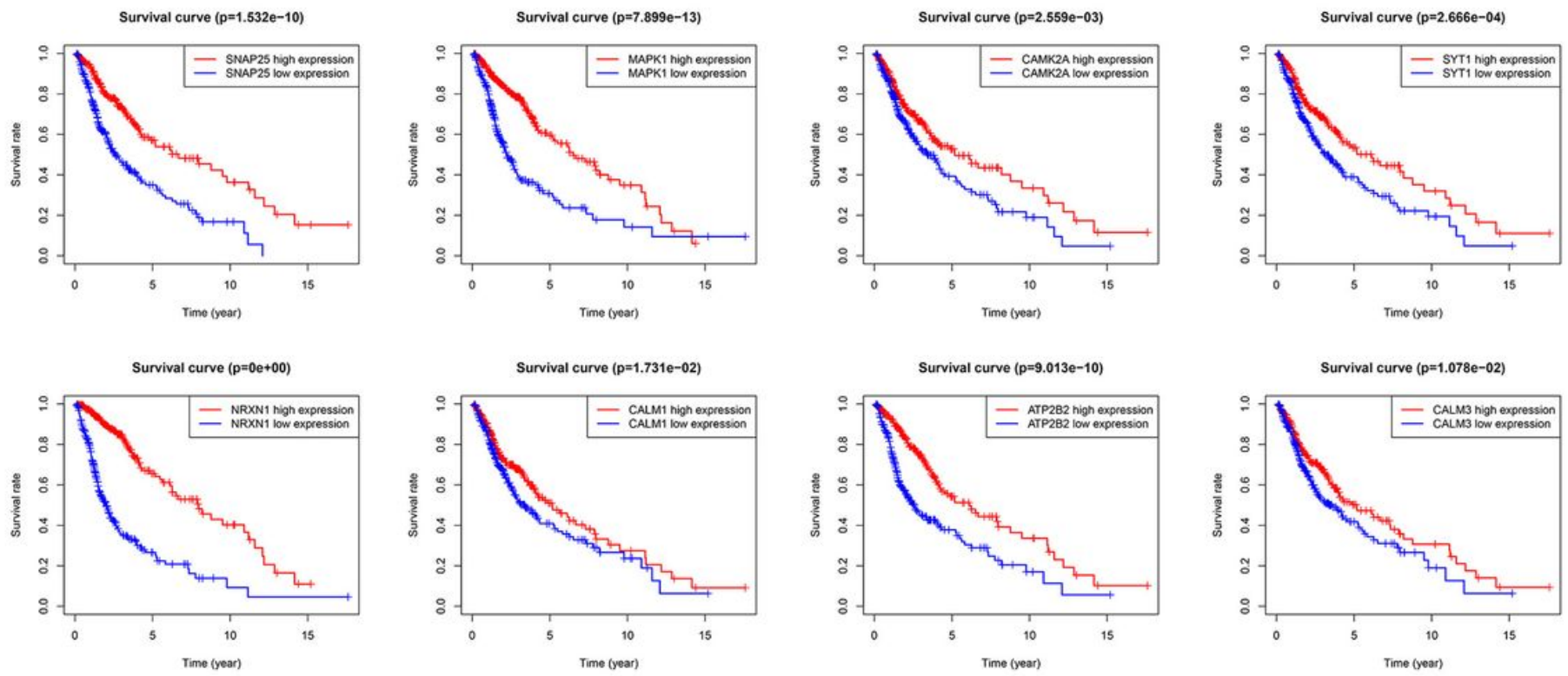

Figure 7

Hub genes associated with survival prognosis through TCGA database.
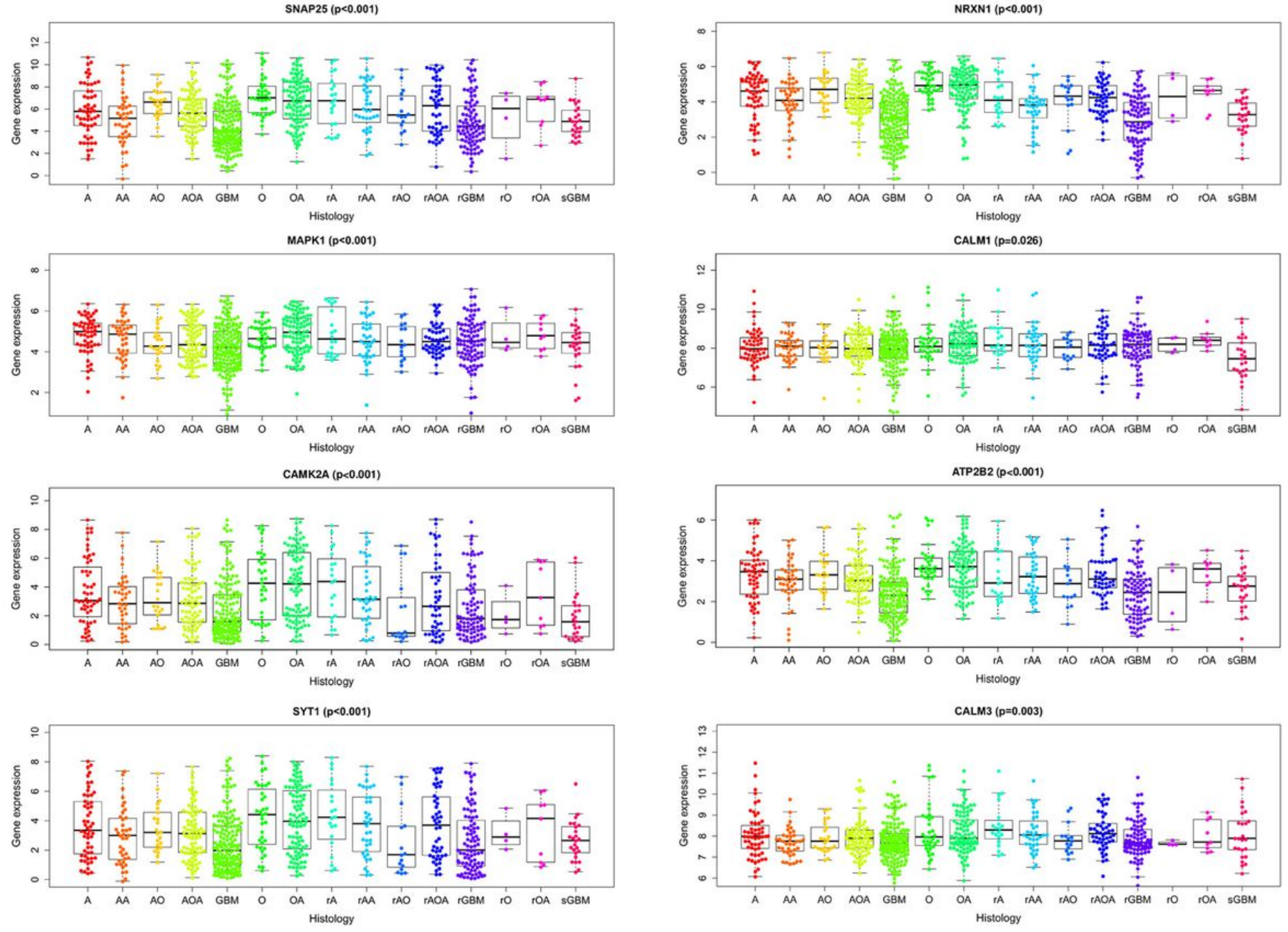

Figure 8 
Relationship between Hub genes and histology through CGGA database. A: astrocytoma; AA: anaplastic astrocytoma; AO: anaplastic oligodendroglioma; AOA: anaplastic oligoastrocytoma; GBM: glioblastoma multiforme; O: oligodendroglioma; OA: oligoastrocytoma; rA: recurrent astrocytoma; rAA: recurrent anaplastic astrocytoma; rAO: recurrent anaplastic oligodendroglioma; rAOA: recurrent anaplastic oligoastrocytoma; rGBM: recurrent glioblastoma multiforme; rO: recurrent oligodendroglioma; rOA: recurrent oligoastrocytoma; sGBM: secondary glioblastoma.

A
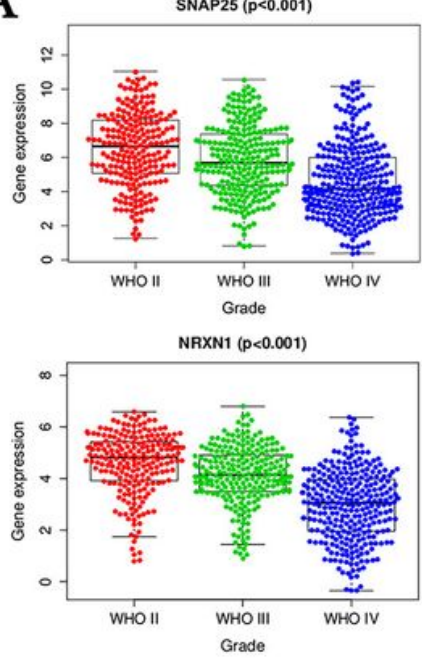

B
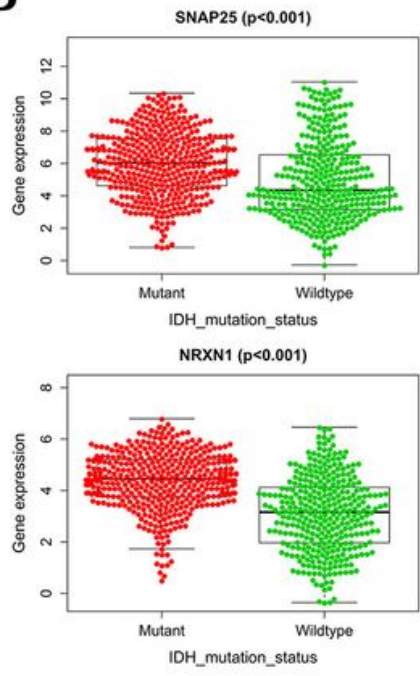

Figure 9
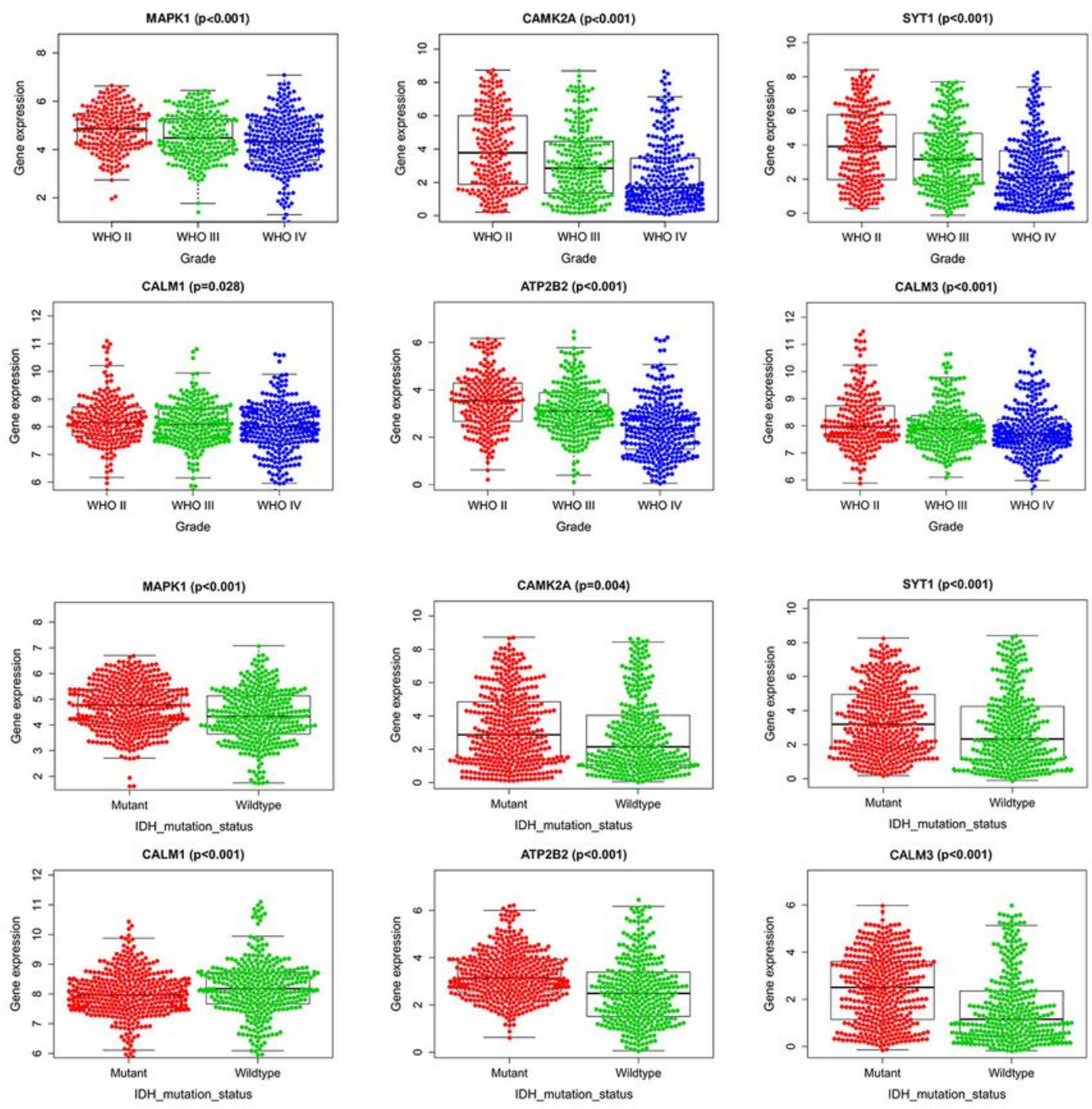

Relationship between Hub genes, grade and IDH mutation through CGGA database. 
SNAP25

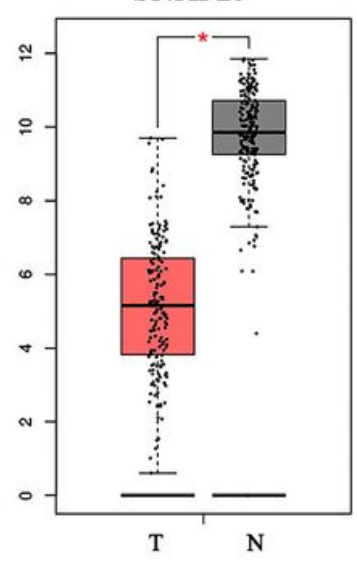

NRXN1

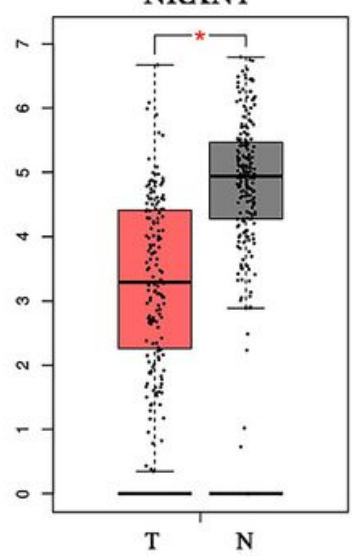

MAPK1



CALM1

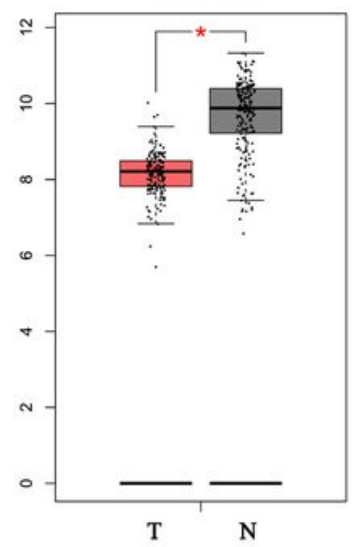

CAMK2A

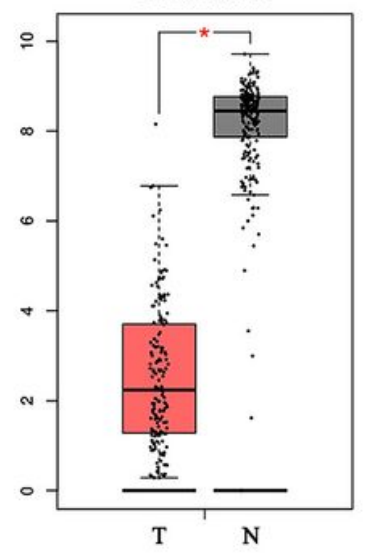

ATP2B2

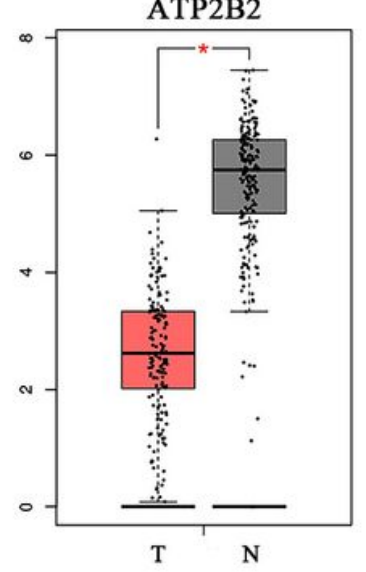

SYT1

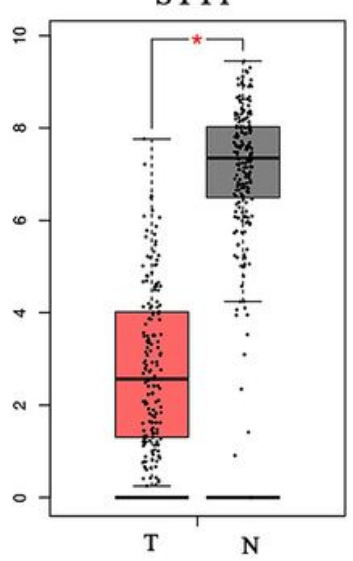

CALM3

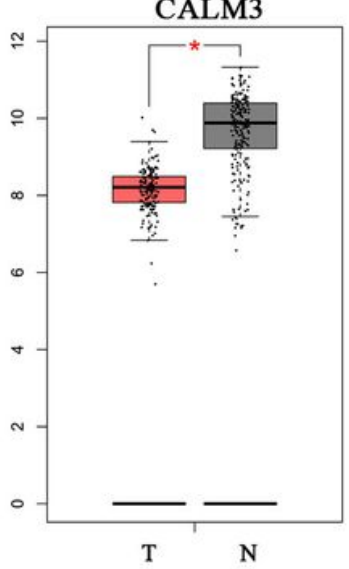

Figure 10

the hub genes expression analysis between the normal and cancer tissues through GEPIA database. Red represent cancer tissues, gray represent normal tissues. ${ }^{\star} \mathrm{P}<0.05,{ }^{\star *} \mathrm{P}<0.01$ vs. Normal tissues.

SNAP25

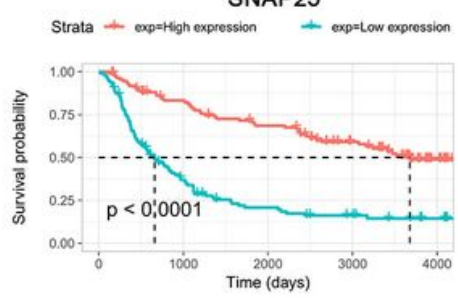

NRXN1



MAPK1

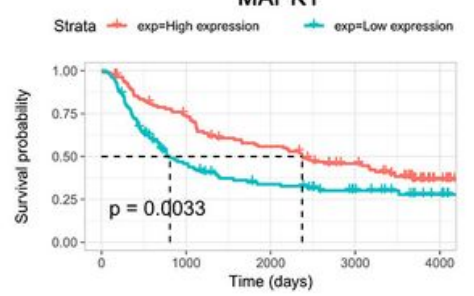

CALM1

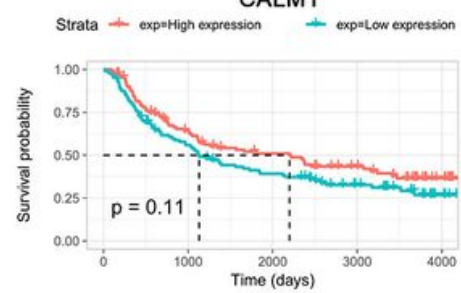

CAMK2A

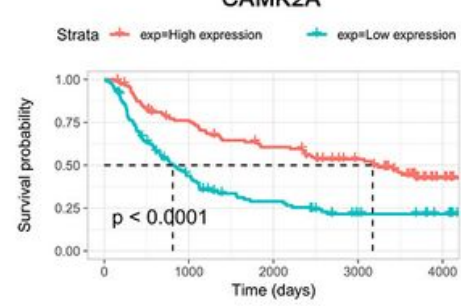

ATP2B2

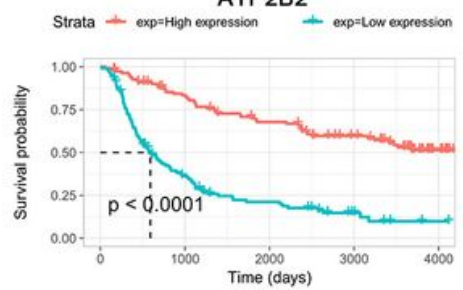

SYT1

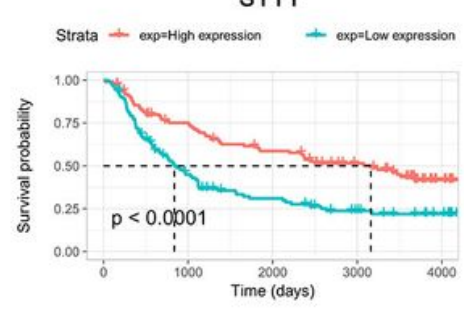

CALM3

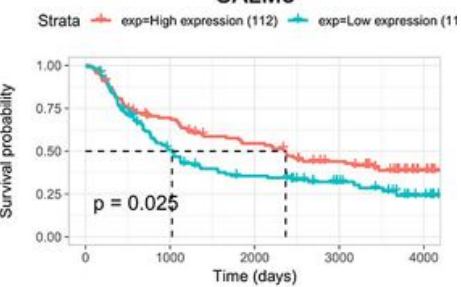

Figure 11

Hub genes associated with survival prognosis through CGGA database. 

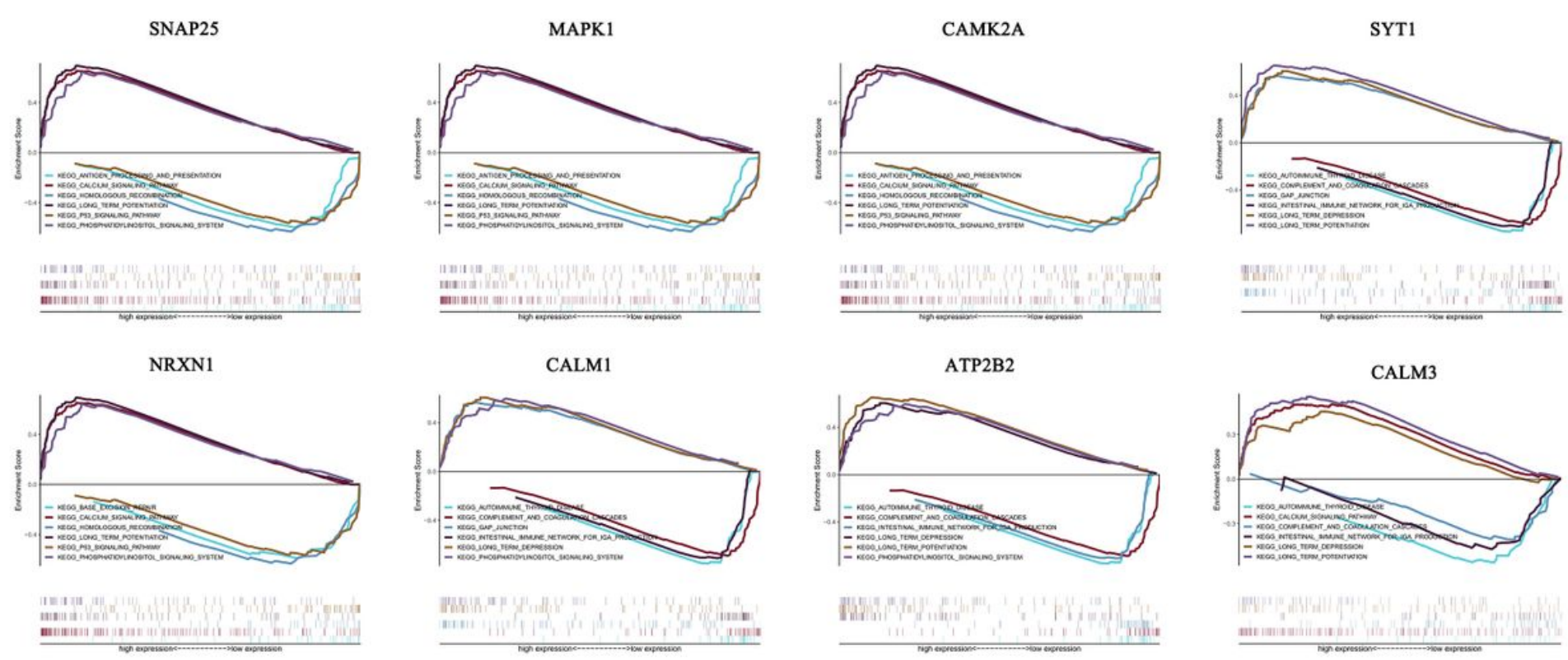

\section{Figure 12}

Investigate the potential functions of thoese hub genes through GSEA.
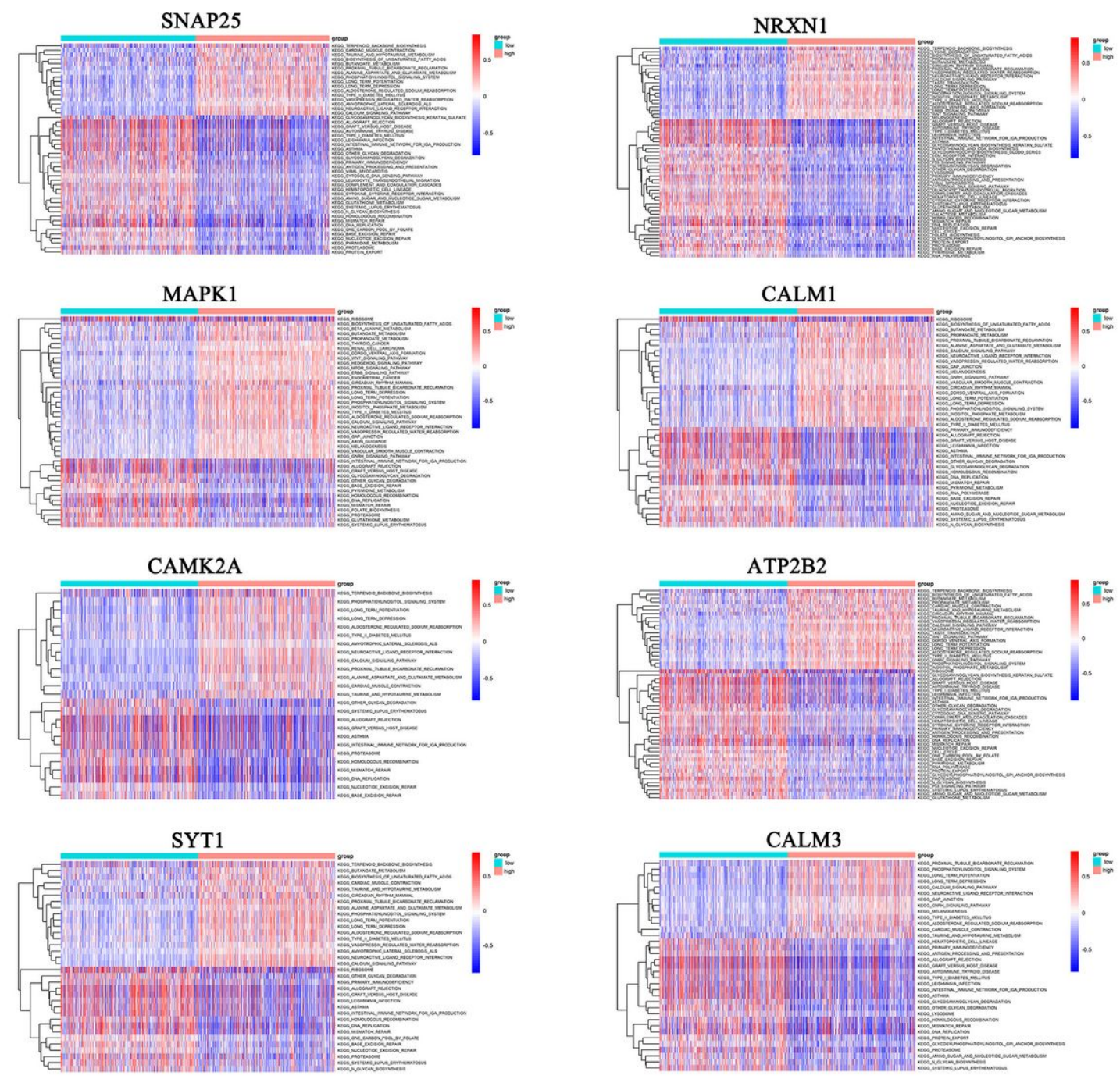

Figure 13 
Investigate the potential functions of thoese hub genes through GSVA.

Page $18 / 18$ 